\title{
La clase y sus tipos como forma de organización esencial de la docencia en la educación superior
}

\section{The class and their types like form of essential organization of the decency in the superior education}

\author{
Enrique Espinoza Freire \\ Universidad Técnica de Machala, Machala, Ecuador \\ eespinoza@utmachala.edu.ec
}

Fecha de recepción: 19/05/2017

Fecha de aceptación: 09/06/2017

Publicado: 24/12/2017

\section{Resumen}

El proceso de enseñanza-aprendizaje en la educación superior tiene varias formas organizativas; independientemente de la importancia que adquiere cada una para el desarrollo de los saberes concebidos en la malla curricular; de las competencias necesarias para determinada profesión, se considera que la clase constituye una de las formas fundamentales de organización de dicho proceso. En la clase, debe lograrse la unidad entre lo instructivo y lo educativo, principio fundamental a tener en cuenta en todo el proceso. El objetivo de este artículo es analizar consideraciones sobre la clase en la educación superior, como forma organizativa esencial en el proceso de enseñanza-aprendizaje, a partir de las características de algunos de sus tipos. En su elaboración, se emplean métodos de investigación del nivel teórico que permiten el procesamiento de la información, la caracterización del objeto de investigación, determinar sus fundamentos teóricos y metodológicos. Los resultados que arroja definen que la clase en la educación superior puede adoptar diferentes tipologías: conferencia, clase práctica, seminario y la clase encuentro, fundamentalmente; cada una tiene características peculiares que permiten tratar conocimientos, desarrollar habilidades y valores, con la participación activa de los estudiantes, lo que favorece su formación integral, meta suprema del sistema educativo. 
Palabras clave: clase, formas de organización de la docencia, enseñanza-aprendizaje, educación superior.

\begin{abstract}
The teaching-learning process in the superior education has several organizational forms; independently of the importance that acquires each one for the development of the knowledge conceived in the curricular mesh, of the necessary competitions for certain profession, it is considered that the class constitutes one in the fundamental ways of organization of this process. In the class, the unit should be achieved between the instructive thing and the educational thing, I begin fundamental to keep in mind in the whole process. The objective of this article is to analyze considerations on the class in the superior education, like form organizational essential in the teaching-learning process, starting from the characteristics of some of its types. In their elaboration, methods of investigation of the theoretical level are used that allow the prosecution of the information, the characterization of the investigation object, to determine their theoretical and methodological foundations. As conclusion, he/she thinks about that the class in the superior education can adopt different tipologías: he/she confers, practical class, seminar and the class encounter, fundamentally; each one has characteristic peculiar that allow to treat knowledge, to develop abilities and values, with the active participation of the students, what favors their integral formation, supreme goal of the educational system.
\end{abstract}

Keywords: class, forms of organization of the teaching, teaching-learning, superior education.

\title{
Introducción
}


El proceso docente presenta diferentes formas organizativas; "se consideran como las principales: la clase, la práctica de estudio, la práctica laboral, el trabajo investigativo, la autopreparación de los estudiantes y la consulta" (MES, 1991). Independientemente de la importancia de cada una para el desarrollo de las materias que conforman el plan de estudio o la malla curricular en determinada carrera, se considera "la clase como la forma fundamental para su organización” (Díaz, 2008; Hernández \& Infante, 2017).

Entendida como "el contexto donde se produce la interacción del docente y de los educandos, y de estos entre sí — sea o no de manera formal en un aula - con el propósito de dar cumplimiento a objetivos trazados" (Hernández \& Infante, 2011), posibilita la formación integral de los estudiantes. El proceso de enseñanza-aprendizaje se materializa, con toda su riqueza y diversidad, en su desarrollo, en cualquier sistema educativo.

En el nivel universitario "se requiere que la clase evidencie la creatividad del docente para motivar a los estudiantes por el estudio" (González, 1990), la ampliación de sus conocimientos, la participación activa en la adquisición y el establecimiento de sus nexos con la futura profesión. Las razones expuestas hacen que en el presente trabajo tome como centro de atención esta forma de organización del proceso, teniendo conciencia de la necesidad de su mejora constante.

\section{La clase como forma organizativa del proceso enseñanza-aprendizaje}

La clase ha sido definida a través de la literatura pedagógica de diferentes maneras. Para algunos como la célula del proceso docente - educativo ya que en ella se presentan íntimamente relacionados todos los componentes del mismo y por otra parte, en ella se funden la instrucción y la educación. Otros enfatizan su carácter ordenado y entonces la aprecian cual forma organizativa en la cual el maestro en un tiempo previamente establecido y en un lugar adecuado a este fin, dirige constantemente la actividad cognoscitiva de sus alumnos, teniendo en cuenta 
particularidades, los medios y los métodos de trabajo necesarios para que los estudiantes se apropien de los conocimientos y desarrollen las habilidades que su nivel de formación les exige.

Una de las cualidades definitorias es que el proceso pedagógico profesional posee un carácter de sistema, donde una vez estructuradas las tareas del profesor y los alumnos, se ejecutan para lograr con calidad los objetivos de los programas docentes mediante la aplicación de los principios didácticos y métodos de aprendizaje, la utilización de los medios necesarios, que contribuyan a la asimilación por los estudiantes, de los contenidos inherentes al objeto de trabajo del profesional, manifestándose en ellos, el crecimiento personal deseado" (Soler, 2003).

Hay definiciones que se centran que la clase es la forma organizativa del trabajo docente diseñada para la eficiente consecución de los objetivos de los planes y programas de estudio mediante la aplicación de los principios didácticos y la utilización de los métodos y medios de enseñanza, cual contribución al mejor desarrollo de este proceso y a la asimilación por los estudiantes de los conocimientos y habilidades inherentes al objeto de trabajo del profesional. Su planificación, preparación, ejecución y control constituye la esencia del proceso docente educativo en la escuela contemporánea.

La clase de nuestros tiempos tiene como premisa fundamental conceder gran importancia a la actividad del estudiante para que forme habilidades y desarrolle potencialidades por medio un proceso de actuación bajo la dirección acertada del maestro. La escuela debe preparar al individuo para que sea capaz de auto educarse y para que además de contemplar y explicar el mundo sea capaz también de transformarlo creadoramente sobre la base de los conocimientos y que sepa tanto alcanzar por sí mismo los conocimientos como renovarlos incesantemente.

La misión de las instituciones educacionales, con independencia del sistema sociopolítico imperante, es propugnar el desarrollo con calidad del proceso docente educativo, logrando una 
adecuada integración de las clases con la actividad investigativa y laboral, así como con las tareas de alto impacto social y demás tareas de carácter extracurricular que cumplen los estudiantes.

Las instituciones de educación superior requieren ofrecer una educación de calidad, por ello se analizan varias investigaciones realizadas en esta década encaminadas a ese fin. "Se define enseñanza de calidad como la que consigue alcanzar las metas de enseñanza, las mismas que se distinguen por su ambición y complejidad como buscar que los alumnos logren un pensamiento crítico, sean creativos y desarrollen habilidades cognoscitivas complejas” (Guzmán, 2011). Sin embargo, de acuerdo con la información disponible, la mayoría de los estudiantes de este nivel no alcanzan esas metas. Se reconoce el papel central que tiene el docente para conseguirlo; por ese motivo, son atendidos las cualidades y dominios que un docente debiera tener. Los estudios analizados se refieren a las buenas prácticas de enseñanza donde abundan ejemplos de investigaciones realizadas en diferentes países a las cuales se critica que, varias de ellas, carezcan de un marco teórico. Al final se aportan algunas sugerencias para mejorar las investigaciones sobre este tema.

A nivel mundial los sistemas de educación superior están siendo sometidos a fuertes presiones para elevar "la calidad de su enseñanza hasta el punto que ésta se ha convertido en su prioridad estratégica” (Cid \& Zabala, 2009 y Hativa \& Goodyear, 2002). Cuestión requerida, entre otras cosas, de una adecuada organización de dicho proceso, toda vez que la docencia no es un acto fortuito y anómalo, sino, por el contrario, "es planificada, organizada y ejecutada científicamente" (Colectivo, 1984; Soler, 2003), según las normas de la Pedagogía y la Didáctica.

Las formas organizativas de la docencia se desarrollan en diferentes escenarios educativos. Cada forma organizativa tiene su propia tipología: 
La clase es una de las formas organizativas del proceso docente educativo, que tiene como objetivos la adquisición de conocimientos, el desarrollo de habilidades y la formación de valores e intereses cognoscitivos y profesionales en los estudiantes, mediante la realización de actividades de carácter esencialmente académico.

Son clasificadas sobre la base de los objetivos que se deben alcanzar y algunos de sus tipos en términos generales son: la conferencia, la clase práctica, el seminario, la clase encuentro, la práctica de laboratorio o de servicio, el taller; aunque en no pocas instituciones de la educación superior son prevalecientes, en dependencia de las exigencias curriculares, las peculiaridades de las carreras que se estudian y del modelo del profesional que se pretende formar, la práctica de estudio, la práctica laboral, el trabajo investigativo de los estudiantes, la autopreparación de los estudiantes, la consulta y la tutoría. Además, se pueden incluir otras formas organizativas en aquellas carreras cuyas particularidades lo justifiquen, en dependencia de las metas de aprendizaje trazadas, o sea, qué se espera que aprendan los estudiantes.

Uno de los rasgos distintivos de este nivel educativo es "buscar resultados de aprendizaje ambiciosos y profundos" (Bermúdez \& Rodríguez, 1996) por ser ya un nivel terminal de estudios, donde concluye la educación formal. Por ejemplo, Hativa (2000) indica que, independientemente del tipo de disciplina o especialidad, las universidades deben formar en sus estudiantes un pensamiento de alto nivel y convertirlos en aprendices autónomos. Si bien estas metas son compartidas, hay diferencias entre las disciplinas acerca de lo que para cada una de ellas es importante. Por ejemplo, los maestros de ciencias y matemáticas le dan gran importancia al aprendizaje del dominio factual de los hechos y principios de sus disciplinas; en cambio, los de humanidades y ciencias sociales otorgan mayor peso al desarrollo personal del estudiante, a la discusión y a las habilidades comunicativas y sociales. 
Por otro lado, Ramsden (2007) clasifica las metas en dos tipos, los cuales se diferencian cualitativamente entre sí. Ellas son: a) las abstractas, genéricas y de desarrollo personal, y b) las referidas al dominio del conocimiento disciplinario incluyendo las habilidades y técnicas particulares que distinguen a cada profesión.

"... la función prioritaria de la universidad es la adquisición imaginativa del conocimiento... Una universidad es imaginativa o no es nada, o por lo menos nada útil" (Whitehead, 1929:139, 145, cit. en Ramsden, 2007: 21); por su parte The Hale Report (1964, cit. en Ramsden, 2007: 21) establece: "Un propósito implícito de la educación superior es lograr que los estudiantes piensen por sí mismos". Metas formuladas más recientemente señalan que "deben aprender cómo aprender" y "pensar críticamente" (Dearing, 1997 cit. en Ramsden, 2007:22). La cualidad del pensamiento crítico es una de las más citadas como meta en este nivel.

En cada modalidad de estudio, el profesor debe utilizar adecuadamente las posibilidades que brinda cada tipo de clase para contribuir al logro de los objetivos educativos formulados en el programa analítico de la asignatura y del año académico en que se desarrolla.

Las cualidades de la clase contemporánea se deducen de las exigencias de desempeñar diversos papeles el profesor de educación superior:

Se espera que sea un excelente maestro, que diseñe cursos y aplique métodos de enseñanza adecuados para cumplir con los requerimientos de una población estudiantil heterogénea, que sepa lidiar con grupos numerosos de estudiantes, que utilice apropiadamente las nuevas tecnologías de la información y la comunicación, que inspire a estudiantes con cero tolerancias a la frustración y cuya mente está más preocupada por su siguiente trabajo de medio tiempo que por el placer de aprender. Al mismo tiempo, se espera que sea altamente productivo en la investigación, que pueda conseguir nuevos recursos financieros, que haga malabares para sortear con las nuevas demandas administrativas y rinda cuentas a una amplia variedad de jefes (Ramsden, 2007: 4-5). 
La clase contemporánea debe cumplir exigencias relacionadas con la educación de la creatividad, la capacidad de observar, de pensar y de generalizar. Presupuestos que elevan el nivel científico y el logro de la profundidad y solidez de los conocimientos de los alumnos. Este logro facilita la educación de la actuación independiente de los alumnos en la actividad cognoscitiva y la estimulación por la auto superación, cualidades sin las cuales se han incumplido el fin de aplicar los contenidos a las habilidades y hábitos en la solución de nuevos problemas. Coinciden varios autores en señalar como se facilita:

La educación de cualidades positivas de la personalidad.

La formación de la cultura laboral.

Atención a las diferencias individuales y el desarrollo de las posibilidades de cada alumno.

La educación del colectivismo en el proceso docente-educativo. (Álvarez de Zayas, 1999; Argudín, 2000):

\section{Breve caracterización de los principales tipos de clase en la educación superior}

Los tipos de clase que mejor se adecuan y responden a las exigencias metas mencionadas (Figura 1) en la enseñanza superior son:

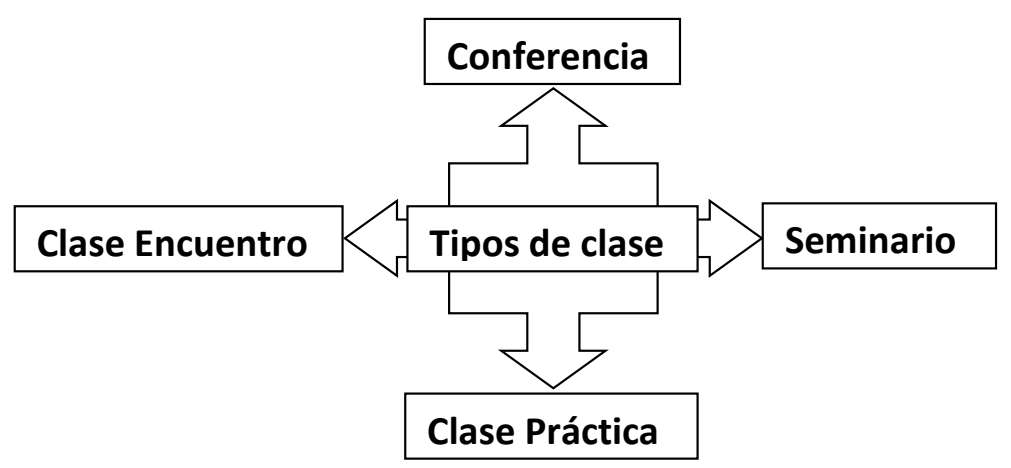

Figura 1. Tipos fundamentales de clase en correspondencia con las exigencias contemporáneas. Fuente: Creación del autor

La conferencia es el tipo de clase que tiene como objetivo instructivo principal la orientación a los estudiantes de los fundamentos científico-técnicos más actualizados de una rama del saber con un enfoque dialéctico-materialista, mediante el uso adecuado de métodos científicos y 
pedagógicos, de modo que les ayude en la integración de los conocimientos adquiridos y el desarrollo de las habilidades y valores que posteriormente deberán aplicar en su vida profesional.

La conferencia básicamente consiste en la exposición por parte del profesor de un contenido científico lógicamente estructurado, en la que este dirige la actividad cognoscitiva y ejerce al mismo tiempo una influencia educativa sobre los estudiantes. Se caracteriza por la amplitud del auditorio y la vía unidireccional de la información. Pueden ser: magistral, orientadoras e informativas.

Se caracteriza por efectuarse para un grupo grande de estudiantes que escuchan al profesor; permitirle al profesor "controlar mediante su estilo de exposición y personalidad las reacciones de los estudiantes y posibilita ejercer una influencia educativa sobre los educandos" (Borgobello, Peralta \& Roselli, 2010, pág. 7).

La estructura de la conferencia incluye tres partes fundamentales y cada una de ellas tiene elementos que son imprescindibles: introducción, desarrollo, conclusiones.

Puede decirse que es utilizada en todo el mundo académico por la importancia de sus funciones (Figura 2).

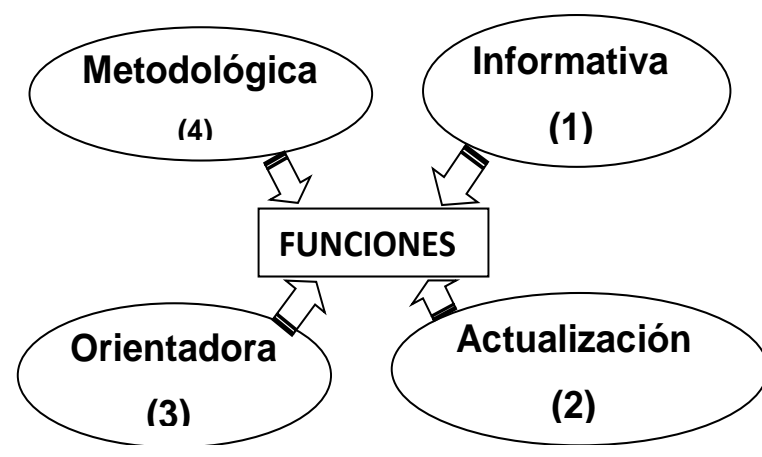

Figura 2. Funciones de la conferencia Fuente: Creación del autor

1. Requiere por parte del profesor una cuidadosa selección del material de su exposición, el cual debe incluir la información fundamental. 
2. Consiste en la necesidad de ofrecer en las conferencias información actualizada, por cuanto los libros, por el tiempo que requiere su publicación no contiene los conocimientos más recientes.

3. Está determinada por la necesidad de guiar a los estudiantes en la búsqueda y estudio de la información complementaria, con el objetivo de ampliar y profundizar los conocimientos.

4. Exige que el profesor al organizar su exposición tenga en cuenta el proceso de aprendizaje de sus alumnos para que además de asimilar el contenido fundamental, se apropien de los métodos de razonamiento.

El seminario se desarrolla para que los estudiantes consoliden, amplíen, profundicen, discutan, integren y generalicen los contenidos orientados; aborden la resolución de tareas docentes mediante la utilización de los métodos propios de la rama del saber y de la investigación científica; desarrollen su expresión oral, el ordenamiento lógico de los contenidos y las habilidades en la utilización de las diferentes fuentes del conocimiento.

Es la forma más propicia para que los alumnos analicen y discutan los problemas objeto de estudio. Es una forma colectiva y activa del proceso docente-educativo. A través de ellos se logra la profundización y sistematización de los contenidos, proporciona amplias posibilidades de desarrollar el pensamiento lógico y abstracto de los estudiantes. Se vincula la teoría con la práctica cotidiana. Desarrollan la habilidad de confección de resúmenes y fichas, así como la expresión oral y escrita.

El seminario se caracteriza por ser una forma colectiva de profundización de los conocimientos que se apoya en el trabajo individual de cada estudiante; la autopreparación y participación resultan imprescindibles para el éxito de la actividad; constituyen el marco idóneo para realizar la retroalimentación de la enseñanza. 
Entre sus funciones principales resaltan la utilización por los estudiantes de métodos y procedimientos racionales de la actividad cognoscitiva: desarrollo en los estudiantes en el empleo de estos métodos, cuya máxima expresión es el método de investigación; la formación de habilidades y hábitos profesionales para la aplicación de conocimientos teóricos; la función de control: Es necesario que el profesor determine que elementos serán objeto del control; el desarrollo de la habilidad de la exposición de los estudiantes y la habilidad para relacionar los conocimientos y aplicarlos.

Para la realización del Seminario se tienen en cuenta las siguientes fases: planificación, preparación, desarrollo, fiscalización y Control

Atendiendo al criterio de algunos autores, los objetivos a lograr y el nivel de asimilación los Seminarios se clasifican de la forma siguiente:

1er Nivel Reproducción: Preguntas y respuestas

Lectura comentada de las fuentes

Taller

2do Nivel. Aplicación:

Problémico

Informe oral y escrito

Debate

Conversación (Este no es necesario evaluarlo)

3er Nivel. Creación:

Mesa Redonda

Conferencia de Prensa

Panel

Ponencia y Oponencia

Simposio 
Integrador

Por su parte, la clase práctica se propone que los estudiantes ejecuten, amplíen, profundicen, integren y generalicen determinados métodos de trabajo característicos de las asignaturas y disciplinas que les permitan desarrollar habilidades para utilizar y aplicar, de modo independiente, los conocimientos.

A partir de ese propósito se han definido cuatro tipos de clase práctica: teórico práctica, ejercitación, laboratorio y reproducción práctica.

La clase práctica se desarrolla siguiendo determinadas fases, a saber, organizativa, introductoria, de ejercitación práctica y final. Es una forma colectiva de desarrollo de las habilidades adquiridas que se apoya en el trabajo individual de cada estudiante. De la autopreparación de los estudiantes depende la efectividad de la actividad. Constituye el marco idóneo para realizar la retroalimentación de la enseñanza.

Tiene funciones muy importantes:

Utilización por los estudiantes de métodos y procedimientos racionales de la actividad cognoscitiva. Desarrolla en los estudiantes el empleo de estos métodos, cuya máxima expresión es el método de investigación.

La formación de habilidades y hábitos profesionales para la aplicación de conocimientos teóricos.

Función de control: Es necesario que el profesor determine qué elementos serán objeto del control.

En las actividades prácticas es importante establecer el control del desarrollo de las habilidades y hábitos, del dominio de los métodos de trabajo, de la vinculación de la teoría con la práctica y de los resultados de la actividad. 
Por último, la clase encuentro consiste en aclarar las dudas correspondientes a los contenidos y actividades previamente estudiados por los alumnos; debatir y ejercitar dichos contenidos y evaluar su cumplimiento; así como explicar los aspectos esenciales del nuevo contenido y orientar con claridad y precisión el trabajo independiente que el estudiante debe realizar para alcanzar un adecuado dominio de los mismos. La misión instructiva más importante que tiene el profesor en la clase encuentro es contribuir al desarrollo de la independencia cognoscitiva de los estudiantes. Es la actividad presencial fundamental en la modalidad semipresencial, aunque puede utilizarse también en la presencial.

Es la clase que se imparte a través de encuentros periódicos entre profesores y estudiantes en horas y lugares previamente establecidos. Dicho encuentro se estructura en tres fases: de control, de orientación y final.

\section{Conclusiones}

Aunque se reconoce la complejidad de la clase, la que debe responder a las metas, exigencias de la carrera, de la materia y, a la vez, a las características de los estudiantes, ante las expectativas que genera en cuanto a su contribución a la formación integral de estos, su importancia en el desarrollo del proceso de enseñanza-aprendizaje es indispensable.

La clase es la forma fundamental de organización de la enseñanza en las disciplinas básicas y básicas específicas, pues constituye la actividad principal en que se materializan planes y programas de estudio. La primera responsabilidad de todo profesor es la de impartir clases de alta calidad. Para ello, resulta una prioridad en el nivel universitario el desarrollo de la tipología que se propone en este artículo, esto es, la conferencia, el seminario, la clase práctica y la clase encuentro, sin menosprecio de las diversas modalidades existentes y otras formas que pedagógicamente son reconocidas, teniendo en cuenta sus características y eficacia para 
conseguir los objetivos formativos que constituyen la misión y el encargo social de las universidades.

\section{Referencias bibliográficas}

Álvarez de Zayas, C. (I999). La escuela en la vida. Didáctica. La Habana: Editorial Pueblo y Educación.

Argudín, Y. (2000). La educación superior para el siglo XXI. Didáctica, Universidad Iberoamericana Santa Fe, 36, 16-25.

Bermúdez, R. \& Rodríguez, M. (1996). Teoría y metodología del aprendizaje La Habana: Editorial Pueblo y Educación.

Borgobello, A., Peralta, N. \& Roselli, N. (2010). El estilo docente universitario en relación al tipo de clase y a la disciplina enseñada. Liberabit, 16 (1), 7-16.

Cid, A. \& Zabalza, M. (2009), "Las prácticas de enseñanza declaradas de los 'mejores profesores' de la Universidad de Vigo", Relieve, 15 (2), 1-29.

Colectivo de autores. (1984). Pedagogía. Editorial. Pueblo y Educación. MINED. ICCP. Ciudad Habana.

Díaz, D. (2008). La clase forma fundamental de organización del proceso pedagógico profesional.

González, A. (1990). Cómo propiciar la creatividad. La Habana: Editorial Ciencias Sociales.

Guzmán, C. (2011). La calidad de la enseñanza en educación superior ¿Qué es una buena enseñanza en este nivel educativo?.

Hativa, N. (2000), Teaching for Effective Learning in Higher Education, Dordrecht/Boston/ London, Kluwer Academic Publishers. 
Hativa, N. \& Goodyear, P. (2002), Teacher Thinking, Beliefs and Knowledge in Higher Education, Dordrecht/Boston/Londres, Kluwer Academic Publishers.

Hernández, R. \& Infante, M. (2011). La relación del estudiante con la identidad: un acercamiento a través de la Literatura local. Ciencias Holguín [Revista Electrónica], XVII (2), 125-140. Recuperado de http://www.ciencias.holguin.cu/

Hernandez, R. \& Infante-Miranda, María Elena. (2017). La clase en la educación superior, forma organizativa esencial en el proceso de enseñanza-aprendizaje. educ.educ. [online]. $20(1), 27-40$.

Ministerio de Educación Superior MES (1991). Reglamento del trabajo docente metodológico en la Educación Superior. La Habana: MES.

Ramsden, P. (2007), Learning to Teach in Higher Education, Londres/Nueva York, Routledge Falmer.

Soler, J. (2003). El proceso pedagógico profesional. Manual de trabajo para el profesor de la enseñanza técnica profesional universitaria. ISPETP. Ciudad Habana. 